\title{
Acinetobacter Baumannii isolates: epidemiology, antibiogram and nosocomial status studied over a 25 month period in a tertiary care hospital in India
}

\author{
N Jaggi', P Sissodia, L Sharma \\ From International Conference on Prevention \& Infection Control (ICPIC 2011) \\ Geneva, Switzerland. 29 June - 2 July 2011
}

\section{Introduction / objectives}

The emergence of Acinetobacter baumannii as an epidemiologically significant nosocomial agent based on its epidemiology, antibiogram patterns and clinical correlation was explored in a 25 month study at a tertiary care hospital in India.

\section{Methods}

The Acinetobacter baumannii isolates over a twenty-five month period (Dec'08-Dec'10) were studied retrospectively for their antibiotic patterns, pathogenic stautus and epidemiology with special reference to nosocomial acquisition.

\section{Setting}

Superspeciality Tertiary care Indian hospital.

\section{Results}

A. baumannii were isolated in 354 samples out of 3036 gram negative isolates (11.6\% prevalence) from the entire hospital in the 25 month period. Maximum isolates were from respiratory secretions (59.8\%) followed by blood (18.6\%). Prevalence of A. baumannii rose to 29.5\% (269 out of 909 gram negatives isolates) in ICU. The nosocomial status of A. baumannii was revealed in its contribution to $39.3 \%$ VAP, $38.7 \%$ CA-BSI, $12.6 \%$ SSI and $16.9 \%$ CA-UTI. Overall resistance of $A$. baumannii for carbapenems was $89 \%$ from all hospital isolates. ICU isolates showed higher resistance (92.9\%) as compared to IPD (83.8\%) and OPD (47.0\%).

\section{Conclusion}

A. baumannii is mainly an ICU bug, showing $75.9 \%$ prevalence (269 isolates out of 354). Overuse of carbapenems in the ICU setting probably led to selection pressure and high level resistance of Acinetobacter to them. Hence implementation of antibiotic policy for judicious use of antibiotics should be stressed on. Also, one must prevent the nosocomial spread of Acinetobacter by appropriate infection control measures.

\section{Disclosure of interest}

None declared.

Published: 29 June 2011

doi:10.1186/1753-6561-5-S6-P291

Cite this article as: Jaggi et al:: Acinetobacter Baumannii isolates:

epidemiology, antibiogram and nosocomial status studied over a 25

month period in a tertiary care hospital in India. BMC Proceedings 20115

(Suppl 6):P291. 